\title{
SCIENTIFIC REP

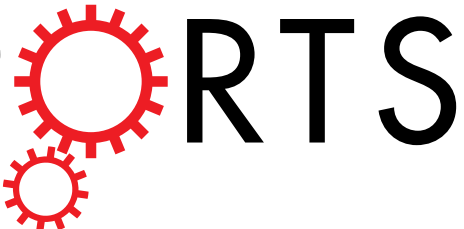

\section{OPEN Thyroid hormone inhibits growth of hepatoma cells through induction of miR-214}

Received: 16 March 2017

Accepted: 5 October 2017

Published online: 01 November 2017
Po-Shuan Huang ${ }^{1}$, Yang-Hsiang Lin ${ }^{1}$, Hsiang-Cheng $\mathrm{Chi}^{2}$, Pei-Yu Chen ${ }^{1}$, Ya-Hui Huang ${ }^{3}$, ChauTing Yeh ${ }^{3}$, Chia-Siu Wang ${ }^{4} \&$ Kwang-Huei Lin ${ }^{1,3,5}$

Thyroid hormone $(\mathrm{TH})$ plays a role in regulating the metabolic rate, heart functions, muscle control and maintenance of bones. 3,3'5-tri-iodo-L-thyronine $\left(\mathrm{T}_{3}\right)$ displays high affinity to nuclear thyroid hormone receptors (TRs), which mediate most TH actions. Recent studies have shown hypothyroidism in patients with an increased risk of hepatocellular carcinoma (HCC). MicroRNAs (miRNAs), a class of non-protein-coding RNA, are suggested to control tumor growth by interacting with target genes. However, the clinical significance of $\mathrm{T}_{3} /$ TR-regulated miRNAs in tumors has yet to be established. In the current study, miRNA expression profile screening was performed using SYBR Green-Based qRTPCR array in TR-overexpressing HepG2 cells. miR-214-3p, which is expressed at low levels in HCC, was stimulated upon $\mathrm{T}_{3}$ application. The $3^{\prime} \mathrm{UTR}$ luciferase reporter assay confirmed that the proto-oncogene serine/threonine-protein kinase, PIM-1, is a miR-214-3p target. PIM-1 was decreased upon treatment with miR-214-3p or $\mathrm{T}_{3}$ stimulation. PIM-1 was highly expressed in $\mathrm{HCC}$, and the effect of PIM-1 on cell proliferation might be mediated by the inhibition of p21. Furthermore, the $\mathrm{T}_{3}$-induced suppression of cell proliferation was partially rescued upon miR-214-3p knockdown. Our data demonstrate that $T_{3}$ induces miR-214-3p expression and suppresses cell proliferation through PIM-1, thus contributing to the inhibition of HCC tumor formation.

Thyroid hormone, 3,3',5-tri-iodo-L-thyronine $\left(\mathrm{T}_{3}\right)$ is a potent mediator of several physiological processes, including embryonic development, cellular differentiation, metabolism and cell growth. Thyroid hormone receptors (TR) are a nuclear receptor superfamily that exert biological functions through transcriptional regulation. Human TRs are encoded by two separate isoform genes, THRA and THRB, which are located on human chromosomes 17 and 3, respectively, and are generated by alternative splicing and different promoter choices. The two genes yield four protein products, designated TR $\alpha 1, \mathrm{TR} \alpha 2, \mathrm{TR} \beta 1$, and $\mathrm{TR} \beta 2^{1} . \mathrm{T}_{3}$ and the TRs regulate gene transcription by binding the thyroid hormone response elements (TREs), which are located in the upstream promoter regions of the target genes. Mutational analyses of rat growth hormone TREs from other $\mathrm{T}_{3}$-responsive genes have led to the identification of a putative consensus hexamer half-site sequence, (G/A)GGT(C/G)A ${ }^{2}$. In particular, TRs bind TREs, in which half-sites are arranged as palindromes $\left(\mathrm{TRE}_{\mathrm{pal}}\right)$, direct repeats (DRs), and inverted palindromes $(\mathrm{IPs})^{2}$. In positively regulated genes, TRs recruit co-repressors to suppress gene transcription in the absence of $\mathrm{T}_{3}$ but release co-repressors and recruit co-activators that stimulate gene transcription in the presence of $\mathrm{T}_{3}{ }^{3}$.

Several controversial studies have been published regarding the relationship between thyroid hormone levels and human cancer ${ }^{4}$. Data from animal models and epidemiologic studies indicate an association between higher thyroid hormone levels and the prevention of liver diseases ${ }^{5,6}$. Dickkopf (DKK) 4, a secreted protein that antagonizes the canonical Wnt signaling pathway, is induced by $\mathrm{T}_{3} / \mathrm{TR}$ at both the mRNA and protein levels in HCC cell lines ${ }^{7} \mathrm{~T}_{3} / \mathrm{TR}$ signaling suppresses cell proliferation by upregulating endoglin, thereby affecting p21 stability ${ }^{8}$. The collective findings suggest that the aberrant expression of $\mathrm{T}_{3} / \mathrm{TR}$ contributes to liver cancer ${ }^{9}$. However, TRs are also implicated in association with MAPK for glioma cells and $\beta$-catenin for intestinal epithelial cells ${ }^{10,11}$.

${ }^{1}$ Department of Biochemistry, College of Medicine, Chang-Gung University, 333, Taoyuan, Taiwan. ${ }^{2}$ Radiation Biology Research Center, Institute for Radiological Research, Chang Gung University/Chang Gung Memorial Hospital, 333, Linkou, Taoyuan, Taiwan. ${ }^{3}$ Liver Research Center, Chang Gung Memorial Hospital, 333, Linko, Taoyuan, Taiwan. ${ }^{4}$ Department of General Surgery, Chang Gung Memorial Hospital, Chiayi, 613, Taiwan. ${ }^{5}$ Research Center for Chinese Herbal Medicine, College of Human Ecology, Chang Gung University of Science and Technology, 333, Taoyuan, Taiwan. Chia-Siu Wang and Kwang-Huei Lin contributed equally to this work. Correspondence and requests for materials should be addressed to C.-S.W. (email: wangcs@cgmh.org.tw) or K.-H.L. (email: khlin@mail.cgu.edu.tw) 
Interestingly, $\mathrm{T}_{3}$ is reported to enhance proliferation in glioma and breast cancer cells ${ }^{12}$, suggesting a dual role of TRs during tumorigenesis in different cancer and disease types.

MicroRNA (miRNAs) are small non-coding RNAs that function in RNA silencing and the post-transcriptional regulation of gene expression ${ }^{13}$. MiRNAs bind the $3^{\prime}$ untranslated region ( $3^{\prime} \mathrm{UTR}$ ) of the mRNA of target genes, which leads to translational repression or mRNA degradation ${ }^{13,14}$. The dysregulation of miRNAs is proposed to be associated with cancer formation. Recent studies have reported that circulating miRNAs serve as potential clinical biomarkers ${ }^{15}$. The functions of miRNAs in tumorigenesis have been investigated in various cancers, including hepatocellular carcinoma (HCC). In the current study, miR-214, which is expressed at low levels in human HCC and is upregulated by $\mathrm{T}_{3} / \mathrm{TR}$, was selected for further analysis. The miR-214 and miR-199 clusters are located on the opposite strands of the Dynamin3 gene (DNM3 $)^{16}$. miR-214 plays an important role in cancer progression and disease severity. Moreover, miR-214 is overexpressed in ovarian and oral mucosal cancers and in malignant melanomas ${ }^{17-19}$. miR-214 inhibits angiogenesis via the suppression of the target gene-HDGF in HCC and is associated with tumor progression and clinical outcome ${ }^{20}$. The expression of miR-214 is significantly associated with $\alpha$-fetoprotein (AFP), which is commonly used as a marker for surveillance in high-risk HCC cases through its presence in serum and other body fluids ${ }^{20,21}$.

PIM-1 is a serine/threonine protein kinase proto-oncogene ${ }^{22}$. The expression of PIM-1 is induced by a variety of growth factors, cytokines, mitogens and hormones. PIM-1 regulates anti-apoptotic activity, cell cycle, and migration through the JAK/STAT pathway ${ }^{23,24}$. Data obtained from clinical studies confirm high expression levels of PIM-1 and support its utility as a prognostic biomarker in prostate cancer, oral cancer, colon cancer, head-and-neck squamous cell carcinoma, and gastric cancer ${ }^{25-29}$. However, the mechanisms underlying PIM-1 signaling in HCC remain to be established ${ }^{30-34}$.

\section{Results}

miR-214 is upregulated by $\mathrm{T}_{3}$. miR-214 was selected for study to demonstrate the potential suppressive role of the TR in view of its downregulation in HCC and positive regulation by $\mathrm{T}_{3} / \mathrm{TR}$. To further ascertain whether $\mathrm{T}_{3}$ /TRs upregulates miR-214 expression in HepG2-TR cells, experiments were performed at various time periods or $\mathrm{T}_{3}$ doses. Notably, the miR-214 level was significantly increased in HepG2-TR $\alpha 1$ and HepG2-TR $\beta 1$ cell lines in a time- and dose-dependent manner. In contrast, miR-214 was only marginally upregulated by $\mathrm{T}_{3} / \mathrm{TR}_{\text {in }}$ HepG2-neo cells devoid of TR expression (Fig. 1a). Further, the host gene, DNM3, was not affected (Fig. 1b), but miR-199a was downregulated by $\mathrm{T}_{3}$ (Fig. 1c). However, two TRE regions $(-5460 \sim-5361$ and $-4560 \sim-4261)$ in the miR-214 upstream promoter were validated and confirmed by the luciferase reporter and chromatin immunoprecipitation (ChIP) assays (Fig. 1d,e).

miR-214 inhibits the proliferation of HCC cell lines. The thyroid hormone suppresses HCC cell proliferation both in vitro and in vivo ${ }^{8}$. To determine whether miR-214 is involved in the $\mathrm{T}_{3} / \mathrm{TR}$-mediated reduction in cell growth, its effect on proliferation was examined. HCC cell lines expressing low (Huh7) and high (SK-Hep1) levels of miR-214 were used for the experiments. Huh7 cells stably overexpressing miR-214 were established (Fig. 2a). Notably, the overexpression of miR-214 in Huh7 cell lines suppressed proliferation and colony formation (Figs 2b,c and S1a). Conversely, SK-Hep1 cells with a stable knockdown of miR-214 were generated, and anti-sense miR-214 expression was detected by via qRT-PCR (Fig. 2d). The depletion of miR-214 led to an enhanced cell proliferation and colony formation in the SK-Hep1 cell line (Figs 2e,f and S1b). Our findings clearly indicate the tumor suppressor role of miR-214 in HCC cell lines.

PIM-1 is the direct target of miR-214. To explore the specific effects of the $T_{3} / T R / m i R-214$ axis in hepatoma cells, the target genes of miR-214 were further investigated. The online database, TargetScan, was used to identify the potential target genes of miR-214, and the luciferase reporter assay performed to validate the regulated genes. Subsequently, the full-length wild-type 3'untranslated region (UTR, WT) and the mutant 3'UTR regions (M1, M2 and M3) of PIM-1 were cloned into a firefly luciferase reporter plasmid, as shown in Fig. 3a. The luciferase activity of the wild-type PIM-1 3'UTR was inhibited by miR-214. Additionally, the M1 mutant (including $\mathrm{M} 1 / 2, \mathrm{M} 1 / 3$ and $\mathrm{M} 1 / 2 / 3$ ) abolished this repression. However, the reporter activity of the M2 or M3 mutant was still repressed by miR-214. HDGF, a known miR-214 target gene, was used as the positive control (Fig. 3a). PIM-1 protein expression was further analyzed via western blot in miR-214 overexpressing and knockdown HCC cell lines. The expression of PIM-1 was clearly suppressed in miR-214-overexpressing cells (Figs 3b and S2a) and, conversely, was increased in SK-Hep1 cells that had been deleted of miR-214 (Figs 3c and S2b).

$\mathrm{PIM}-1$ is a proto-oncogene in $\mathrm{HCC}$ that is regulated by $\mathrm{T}_{3}$. PIM-1 was identified as a proto-oncogene in many cancers, including pancreatic, prostate, gastric and skin cancer and leukemia ${ }^{25,35-38}$, but its underlying mechanism of action in HCC remains to be established. The effects of $\mathrm{T}_{3} / \mathrm{TR}$ on PIM-1 expression were determined in HepG2-TR cells. Our data showed that PIM-1 expression is decreased in HepG2-TR $\alpha 1$ and HepG2-TR $\beta 1$ cell lines (Figs 3d and S3a). The stable depletion of PIM-1 was achieved and detected via western blot (Figs $3 e$ and S3b,d). The knockdown of PIM-1 suppressed cell proliferation and colony formation (Figs $3 \mathrm{f}$ and $\mathrm{S} 3 \mathrm{c})$, supporting its role as a proto-oncogene in HCC.

miR-214 suppresses cell proliferation by modulating the PIM-1 pathway. To address the possible involvement of PIM-1 in the miR-214-mediated suppression of cell growth, PIM-1 was re-expressed in miR-214-overexpressing Huh7 cells (Figs 4a and S4a). Interestingly, the overexpression of miR-214 in Huh7 cells inhibited their cell proliferation capacity (Fig. 4b), which was rescued following the re-expression of PIM-1 (Fig. 4b). p21 is inhibited by PIM-1 in other cancer types, but its expression patterns in HCC are currently unclear. SK-Hep1 and HepG2-TR $\alpha 1$ cell lines with the miR-214 knockdown were established. Upon the suppression of miR-214, the expression of PIM-1 increased, and that of p21 decreased (Figs 4c and S4b). These findings 
(a)

HepG2-TR-neo_miR-214

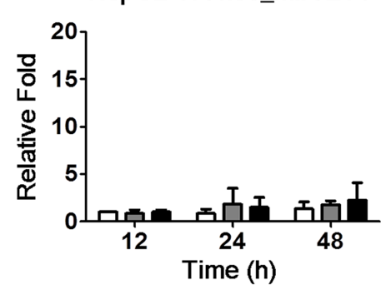

(b)

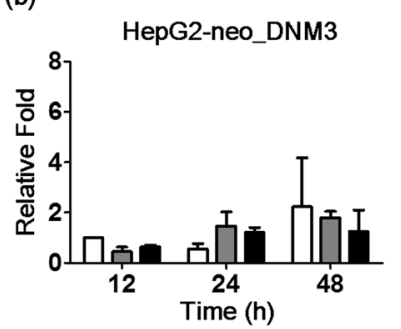

(c)

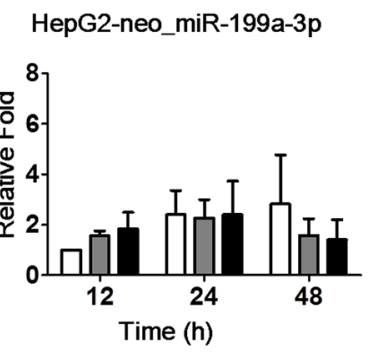

(d)

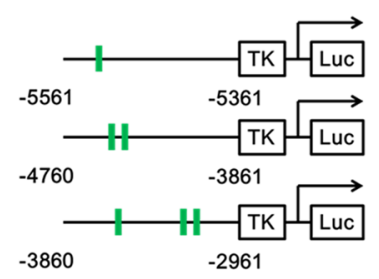

| Pal:AGGTCATGACCT
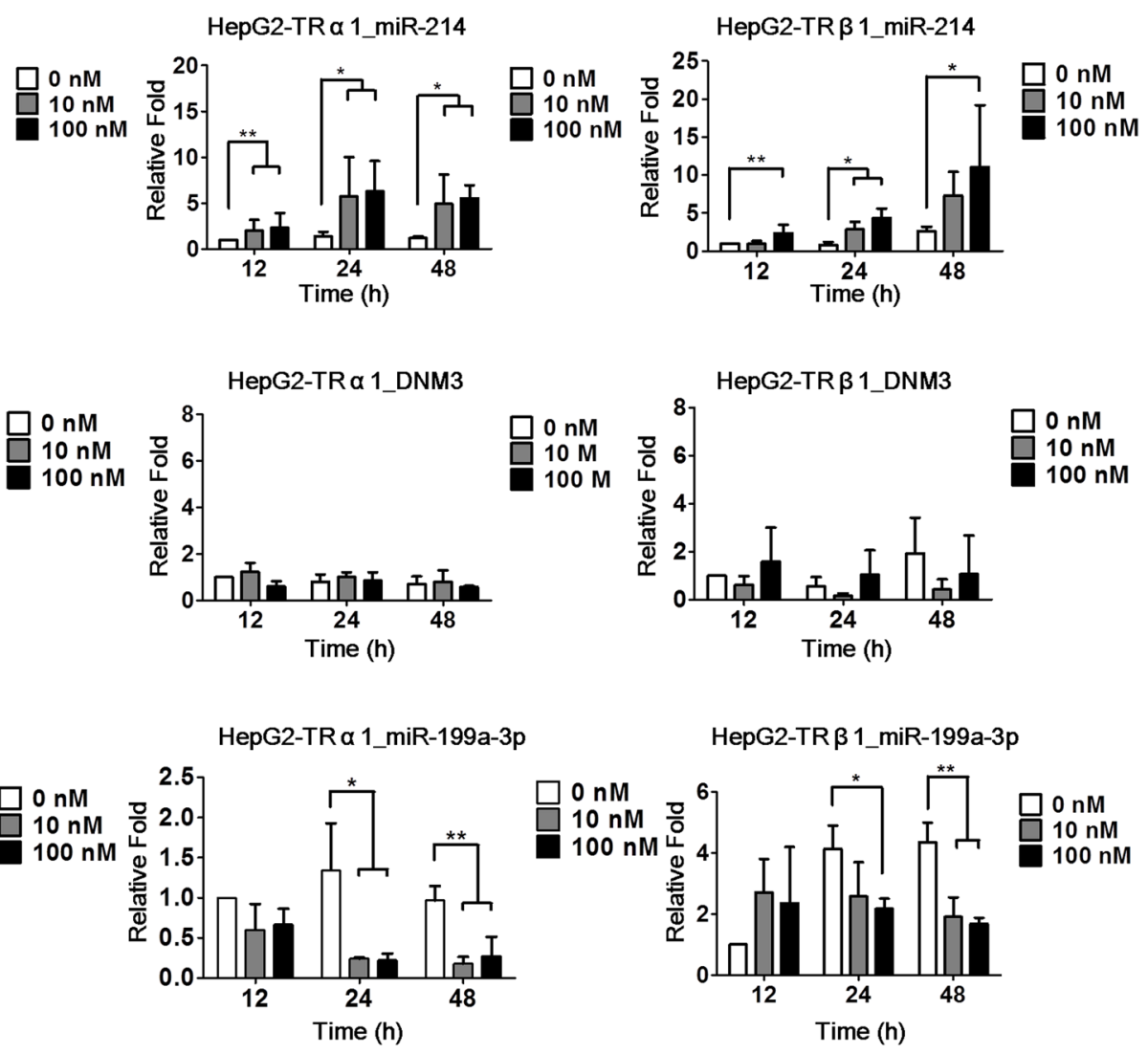

(e)
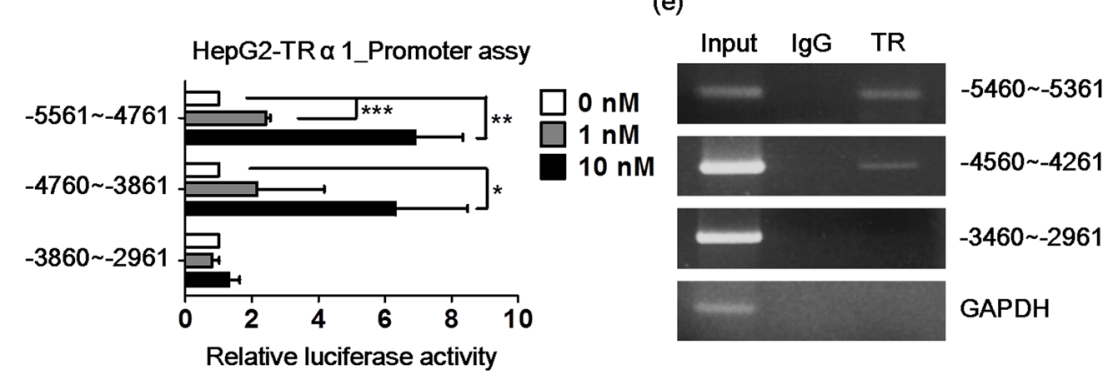

Figure 1. miR-214 is upregulated by $\mathrm{T}_{3}$. HepG2-TR cell lines were treated with $0-100 \mathrm{nM} \mathrm{T}_{3}$ for $12-48$ hrs. (a) miR-214, (b) DNM3 and (c) miR-199a-3p were measured by qRT-PCR. U6 was used as a loading control. (d) HepG2-TR $\alpha 1$ cells were transfected with miR-214 reporter plasmids. The cells were incubated for $24 \mathrm{hrs}$ with thyroid hormone $(0-10 \mathrm{nM})$. (e) A ChIP assay was used to confirm that TR was directly bound to the miR214 promoter region. GAPDH was the negative control. The data are presented as the means \pm s.d. of three independent experiments $(* \mathrm{p}<0.05 ; * \mathrm{p}<0.01 ; * * \mathrm{p}<0.001$ vs. $12 \mathrm{hrs}, 0 \mathrm{nM})$.

suggest that miR-214 suppresses cell proliferation by modulating the PIM-1 pathway and that PIM- 1 is a direct target gene of miR-214 in hepatoma cells.

Thyroid hormone suppresses cell proliferation through the upregulation of miR-214. To ascertain whether $\mathrm{T}_{3}$ regulates PIM-1 through the induction of miR-214, miR-214 expression was depleted in HepG2-TR $\beta 1$ cell lines. The expression of the PIM-1 protein was suppressed by $\mathrm{T}_{3} / \mathrm{TR}$, which was abolished upon miR-214 knockdown (Fig. 4d), indicating that $\mathrm{T}_{3}$ upregulates miR-214 and suppresses PIM-1 protein. Notably, the $\mathrm{T}_{3}$-induced inhibition of cell proliferation was restored upon the knockdown of miR-214 (Figs 4e and S4c). Based on the collective results, we propose that $\mathrm{T}_{3}$ suppresses cell proliferation through the miR-214-mediated repression of PIM-1.

miR-214 reduces tumor formation in vivo. To determine whether the in vitro effects of miR-214 can be replicated in vivo, xenograft mouse models were used. As expected, the overexpression of miR-214 in the Huh7 
(a)

Huh7_miR-214 expression

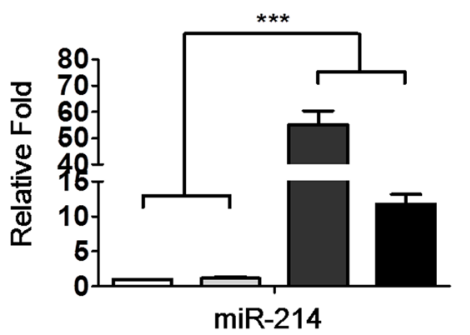

(c)

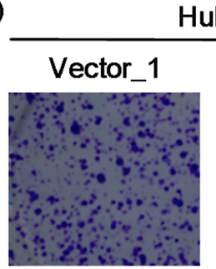

miR-214_1

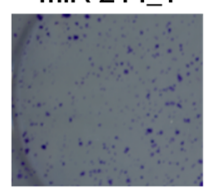

(e)

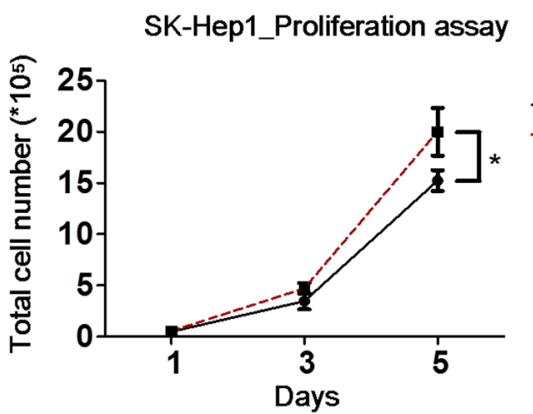

(b)

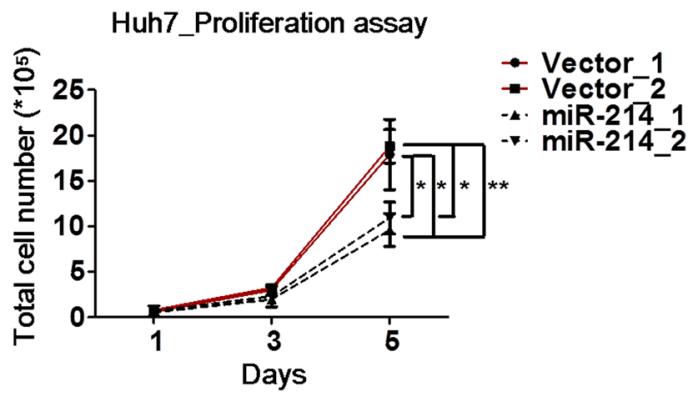

(d)

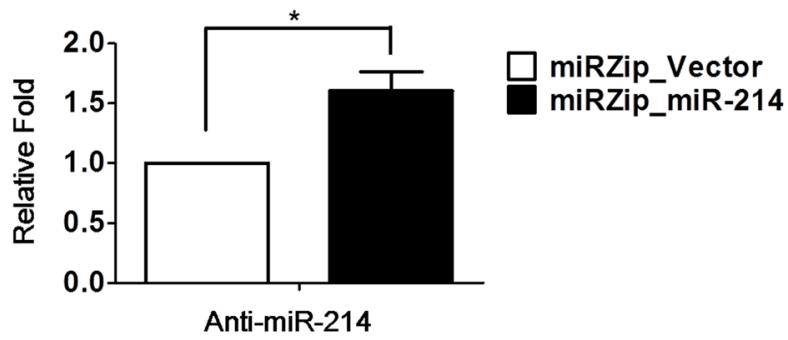

(f)

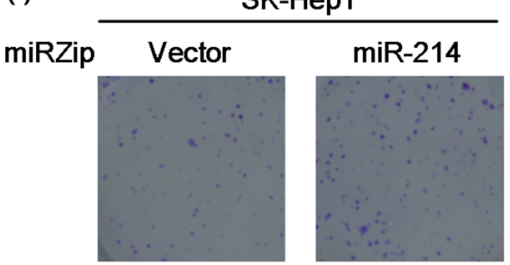

Figure 2. miR-214 inhibits the proliferation of HCC cell lines. Huh7 cell lines stably expressing miR-214 were established by transfection with the miR-214 plasmid. (a) The expression levels of miR-214 were measured by qRT-PCR and the vector pcDNA6.2 was used as a control, respectively. (b) The Huh7 hepatoma cell line proliferation capacity was measured by the total cell numbers. (c) Colony assay in the Huh7 cells. (d) The stable knockdown of miR-214 in the SK-Hep1 cell lines was established by infection with the lentivirus miRZipmiR-214 (Anti-miR-214). The expression levels of anti-miR-214 were measured by qRT-PCR. U6 was used as a loading control. (e) The SK-Hep1 hepatoma cell lines proliferation capacity was measured. (f) The colony assay in SK-Hep1. The data are presented as the means \pm s.d. of three independent experiments $(* \mathrm{p}<0.05$; $* * \mathrm{p}<0.01 ; * * * \mathrm{p}<0.001$ vs. control vector).

cell line led to the inhibition of tumor formation compared with the control group (Fig. 5a). Previously, to generate hyperthyroid $\mathrm{HBx}$ mice, $\mathrm{T}_{3}(2.5 \mathrm{mg} / \mathrm{L})$ was added to the drinking water for 8 months. To induce hypothyroidism in mice, $0.02 \%$ methimazole plus $0.1 \%$ sodium perchlorate was added to the drinking water for 6 months ${ }^{39}$. Clearly, the data indicate that miR-214 was upregulated (Fig. 5b, upper panel) and that the target PIM-1 protein (Fig. 5b, lower panel) was downregulated by $\mathrm{T}_{3}$ in vivo. Additionally, in the mice treated with diethylnitrosamine (DEN) combined with a choline-deficient diet for weeks, multiple preneoplastic lesions were observed ${ }^{40}$. Notably, $\mathrm{T}_{3}$ plays a suppressor role to inhibit DEN-induced HCC, which is probably mediated by the upregulation of miR214 (Fig. 5c, upper panel) to repress the target gene PIM-1 (Fig. 5c, lower panel) at the protein level.

The expression of miR-214 in clinical specimens was measured via qRT-PCR. The expression of miR-214 was lower ( $\mathrm{p}<0.0001$, Fig. $5 \mathrm{~d}$ ), whereas that of PIM-1 was higher in the HCC tumors compared with the adjacent normal tissues (Fig. 5e); the inverse correlation is observed between PIM-1 and miR-214 (Fig. 5f). Moreover, PIM-1 expression was negatively correlated with TR (Fig. 5g). Our results further validate the tumor suppressor role of miR-214 in vivo. Additionally, the thyroid hormone suppresses HCC cell proliferation by inducing miR214 to repress PIM-1(Fig. 5h). 
(a)

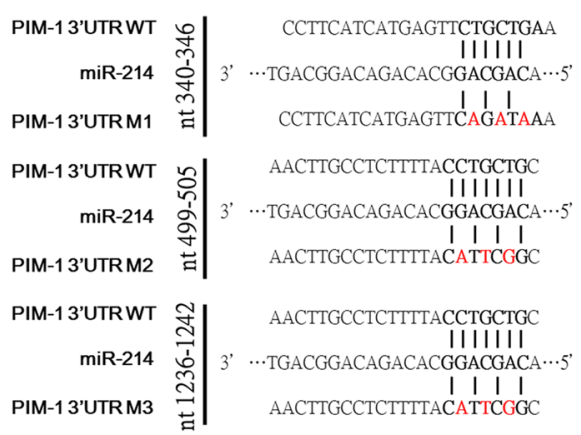

(b)

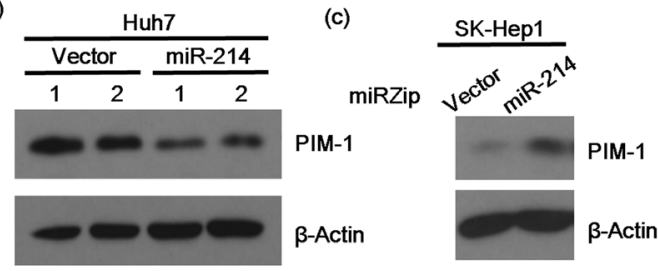

(e)

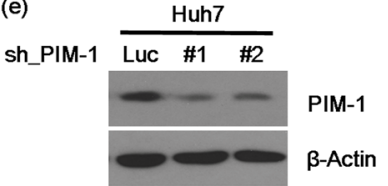

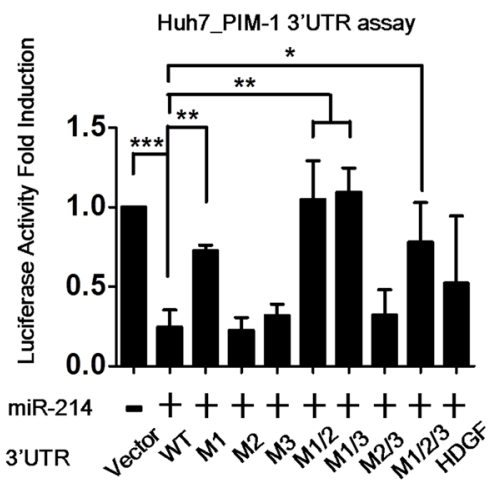

(d)

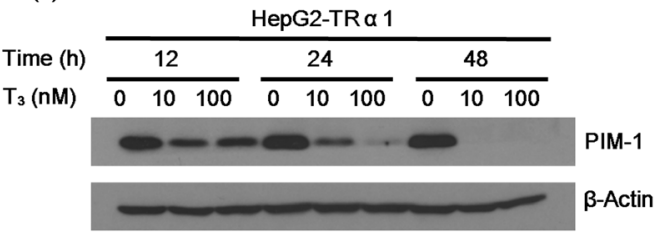

Time $(\mathrm{h})$

$\mathrm{T}_{3}(\mathrm{nM})$

(f)

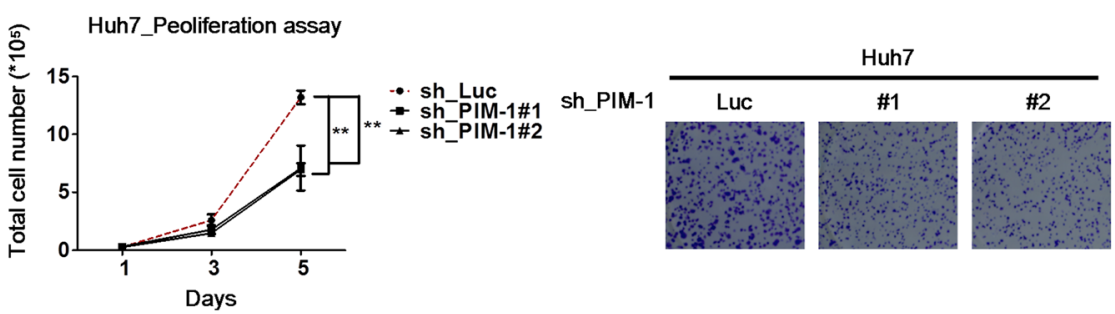

Figure 3. PIM-1 is the direct target of miR-214 and is regulated by $\mathrm{T}_{3}$ in HCC. (a) The wild-type (WT) and mutant (M1-M3) 3'UTRs of PIM-1 were cloned, and (left) the luciferase reporter assay was performed in the Huh7 cell line (right) that stably expressed miR-214 or the vector control. (b) PIM-1 protein levels in the Huh7 stable expressing and (c) SK-Hep1 stable knockdown miR-214 cells was measured by western blot. (d) The HepG2-TR cell lines were treated with 0-100 $\mathrm{nM} \mathrm{T}_{3}$ for 12-48 hrs, and PIM-1 protein levels were measured by western blot. (e) The stable knockdown PIM-1 in the Huh7 cell lines was established by transfection with the shRNA PIM-1 plasmids. (f) The Huh7 hepatoma cell line proliferation capacity was measured by a proliferation assay or a colony formation assay. $\beta$-actin was used as a loading control. The data are presented as the means \pm s.d. of three independent experiments $\left({ }^{*} \mathrm{p}<0.05 ; * * \mathrm{p}<0.01\right.$; ***p $<0.001$ vs. Vector), and the luciferase activity was normalized with beta-galactosidase.

\section{Discussion}

In this study, we showed that miR-214, regulated by $\mathrm{T}_{3} / \mathrm{TR}$, acts as a tumor suppressor in HCC cell lines. A previous study indicated that Twist1 interacts with the E-box of the miR-214/199a promoter region to stimulate the expression of the host gene DNM3os, encoding miR-214 and miR-199a in neural cells ${ }^{41}$. However, miR-199a expression was not upregulated by $\mathrm{T}_{3} / \mathrm{TR}$. The differential regulation is due to TREs in the region between miR199a and miR-214. Similarly, the miR-17-92 cluster containing six miRNAs is processed from the transcript of $\mathrm{C} 13$ orf25 in lymphomas and solid tumors, but the expression is variable. These findings suggest that the processing or stability of miRNAs is differentially regulated ${ }^{42,43}$.

Several research groups have reported that the overexpression of miR-214 suppresses cell proliferation. miR214 inhibits the expression of E2F2, CDK3 and CDK6 in HCC ${ }^{44}$. The reduced expression of miR-214, via the activation of the hepatoma-derived growth factor (HDGF), is observed in $\mathrm{HCC}^{20,44}$. miR-214 is additionally downregulated in colorectal cancer through promoter hypermethylation ${ }^{45}$. Lu et al. ${ }^{46}$ demonstrated that miR-214 downregulates UDP-N-acetyl- $\alpha$-D-galactosamine: polypeptide $\mathrm{N}$-acetylgalacto- saminyltransferase 7 (GALNT7) protein expression to suppress the invasive ability of esophageal squamous cell carcinoma. Furthermore, miR-214 
(a)

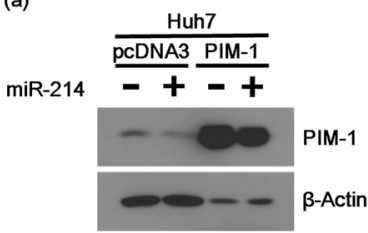

(b)

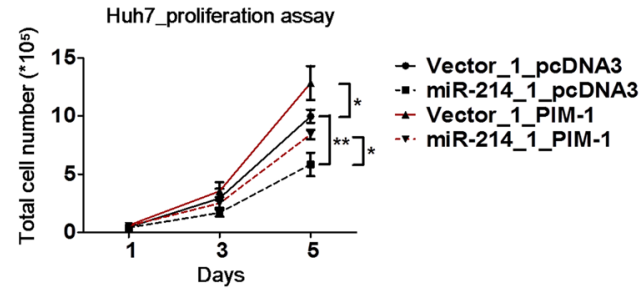

(c)

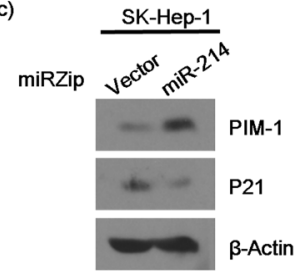

(e)
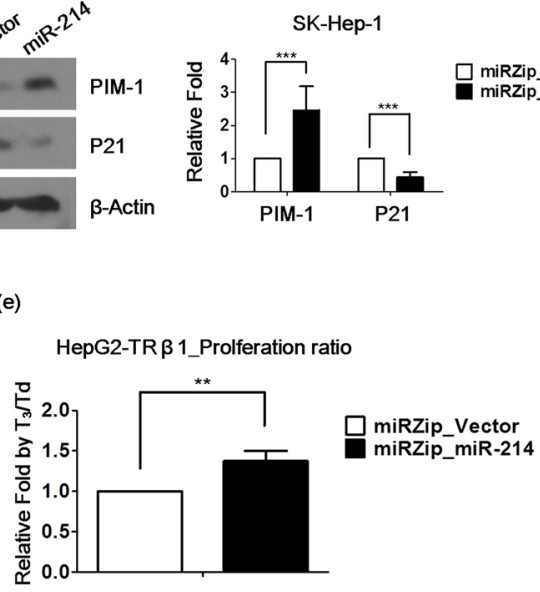

(d)
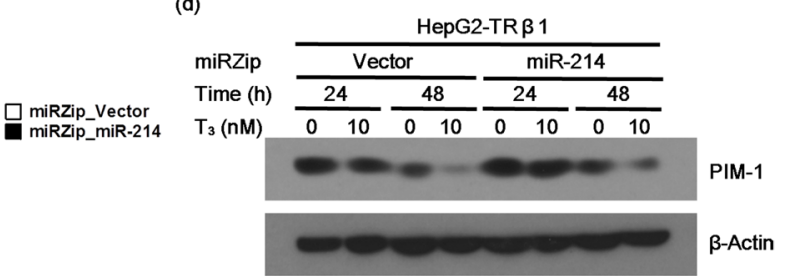

HepG2-TR 31 PIM-1

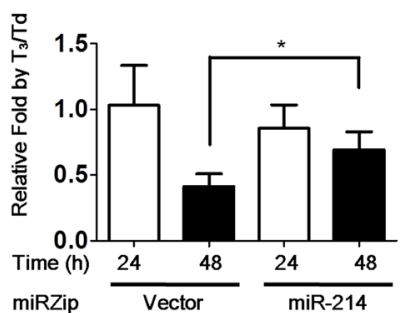

Figure 4. Thyroid hormone suppresses cell proliferation through the upregulation of miR-214 by modulating the PIM-1 pathway. (a) The re-expression of PIM-1 in the miR-214 stable Huh7 cell lines, and the PIM-1 protein levels were measured by western blot. (b) The proliferation capacity of the Huh7 hepatoma cell lines was measured by a proliferation assay or the colony formation assay with/without PIM-1 re-expression. (c) PIM-1 and p21 protein expression were measured in stable knockdown miR-214 SK-Hep1 or HepG2-TR $\alpha 1$ cell lines by western blot. (d) HepG2-TR $\beta 1$ depleted miR-214 cell lines were treated with $0-10 \mathrm{nM} \mathrm{T}_{3}$ for 24-48 hrs, and the PIM-1 protein levels were measured by western blot. $\beta$-actin was used as a loading control. The PIM- 1 protein levels are shown by the relative fold of $\mathrm{T}_{3}$ depleted $(\mathrm{Td}) / \mathrm{T}_{3}$. (e) HepG2-TR $\beta 1$ depletion of the miR-214 cell lines were treated with $0-10 \mathrm{nM} \mathrm{T}_{3}$, and the proliferation capacity was measured and is shown by the relative fold change of the $\mathrm{Td} / \mathrm{T}_{3}$ ratio. The data are presented as the means \pm s.d. of three independent experiments (*P $<0.05 ; * * \mathrm{P}<0.01 ; * * * \mathrm{P}<0.001$ vs. Vector).

mediates the downregulation of HMGA1 to inhibit growth and motility in human cervical and colorectal cancer cells $^{47}$. The collective results are consistent with our finding that miR-214 plays a tumor suppressor role.

Conversely, the oncogenic role of miR-214 in osteosarcoma is reported ${ }^{48}$. miR-214 binds directly to the $3^{\prime} \mathrm{UTR}$ of LZTS1 mRNA to suppress its expression at both the transcriptional and translational levels, thus promoting osteosarcoma cell proliferation, invasion and tumor growth ${ }^{49}$. Alimirah and co-workers reported that a vitamin D receptor suppressed miR-214 but that the overexpression of miR-214 activated the hedgehog pathway to promote breast cancer progression. miR-214 acts as an oncogene in breast cancer to promote cell invasion through the downregulation of P53 $3^{50,51}$. Moreover, miR-214 enhances the peritoneal metastasis of gastric cancer cells through the downregulation of PTEN expression ${ }^{52}$. However, miR-214 is downregulated by $\mathrm{T}_{3} / \mathrm{TR}$ in the mouse heart. Myocardial infarction (MI)-induced cardiac stress results in the activation of Dio3, leading to a reduced level of cardiac $\mathrm{T}_{3}$, which in turn, stimulates miR-214 expression ${ }^{53}$. The role of miR-214 in cardiac fibrosis, whereby miR-214 induces proliferation and collagen synthesis to contribute to cardiac injury, is not consistent with our finding in $\mathrm{HCC}^{54}$. We speculate that miRNA-214, which is regulated by $\mathrm{T}_{3}$, occurs in a tissue-specific manner. $\mathrm{T}_{3}$ induces cell proliferation to promote organ development in muscle, bone and heart ${ }^{55-57}$. However, according to our previous report, $\mathrm{T}_{3}$ represses cell proliferation in liver cancer cells $\mathrm{s}^{58}$.

PIM-1 is the miR-214 target gene and is overexpressed in many cancer types, including prostate and breast cancers ${ }^{22,59-62}$. In our experiments, the PIM-1 protein levels were upregulated in the HCC specimens. The depletion of PIM-1 in HCC cell lines led to the suppression of the proliferation rate, suggesting that PIM-1 acts as a proto-oncogene in HCC. PIM-1 was further confirmed as a miR-214 target gene, consistent with an earlier study showing that is PIM-1 is a target gene of miR-214 in mesothelioma ${ }^{63} \cdot$ p2 $^{2} 1$ is one of the downstream gene targets of PIM-1 in prostate, colon and lung carcinoma ${ }^{64-66}$. Here, the knockdown of miR-214 in HCC cell lines induced PIM-1 and the consequent inhibition of $\mathrm{p} 21$ expression. A previous study demonstrated that $\mathrm{T}_{3} / \mathrm{TR}$ suppresses cell proliferation by upregulating endoglin controlling p 21 stability ${ }^{8}$. We propose that $T_{3} / T R$ regulates miR-214 expression to reduce PIM-1 expression and enhance 21 expression. 

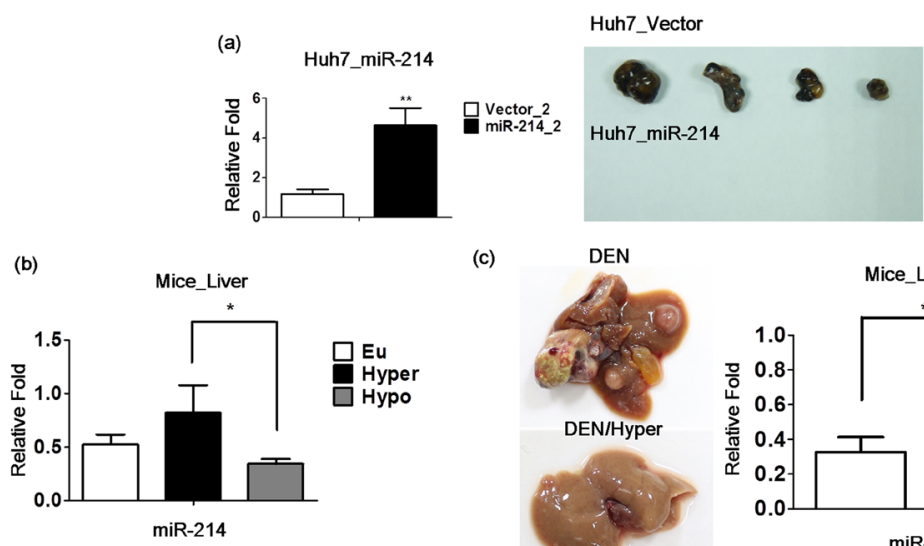

(c)
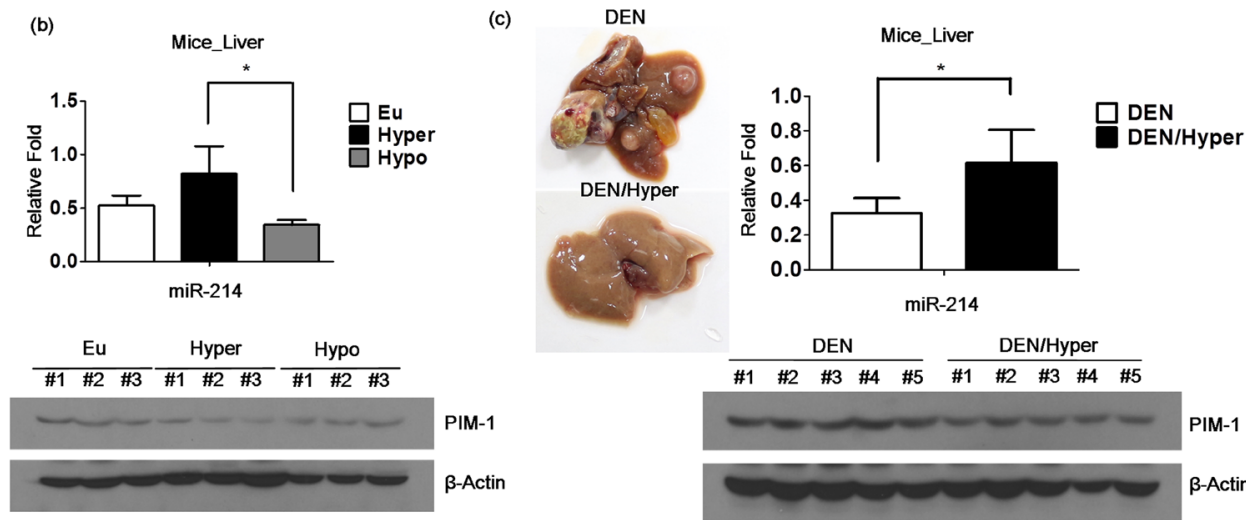

Mice_Liver

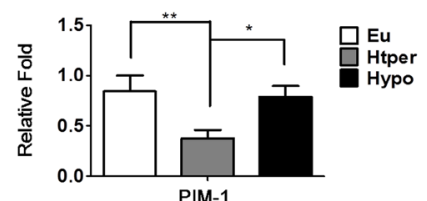

(d) HCC specimens miR-214

(e) HCC specimens_PIM-1 Protein (f) Correlation of miR-214/ PIM-1
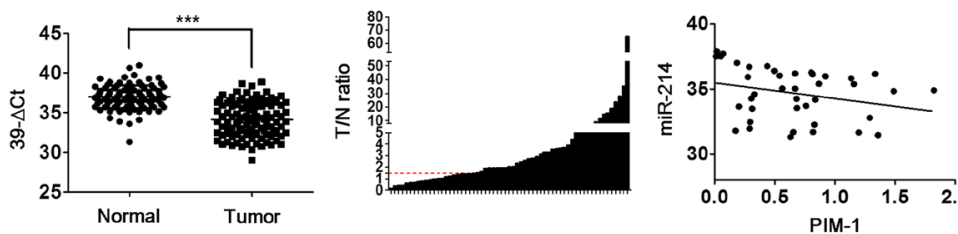

Spearman:-0.341

(g)

$\mathrm{P}<0.0001$

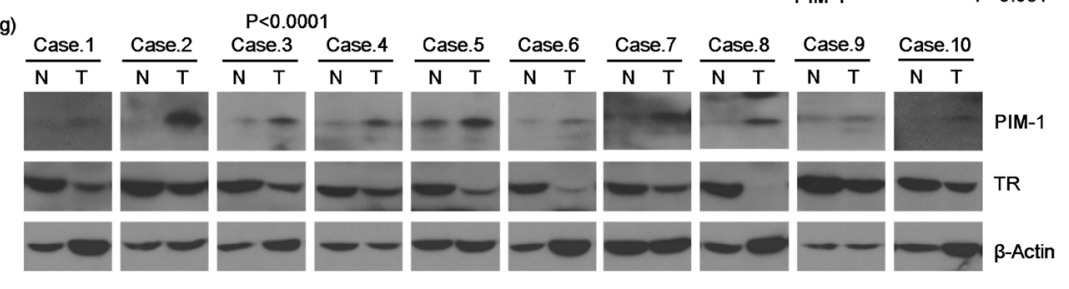

(h)

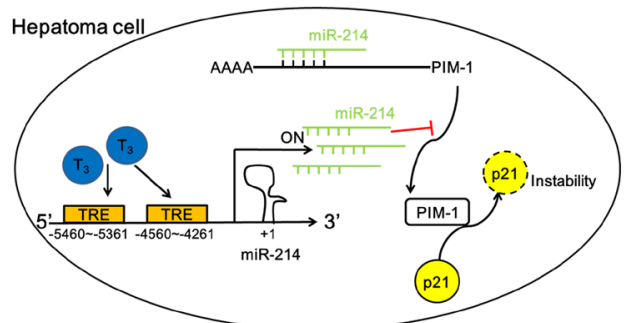

Figure 5. miR-214 reduces tumor formation in vivo. (a) Stable expressing miR-214 and the control (pcDNA6.2) Huh7 cell lines were subcutaneously injected into nude mice. (b) miR-214 expression was detected in the mice livers ( $\mathrm{n}=3$ ) with Euthyroid (Eu), Hypothyroid (Hypo), and Hyperthyroid (Hyper) by qRT-PCR. (c) To detect miR-214 expression in the mice liver $(\mathrm{n}=5)$ with DEN or DEN/Hyper, and the morphology of the livers, and the U6 was a loading control. The data are presented as the means \pm s.d. $(* \mathrm{p}<0.05$; $* * \mathrm{p}<0.01 ; * * * \mathrm{p}<0.001$ vs. Hypo or DEN). (d) miR-214 expression levels were determined in 108 pairs of HCC specimens by qRT-PCR, and U6 was used as a loading control. The data are presented as 39- $\Delta \mathrm{Ct}$ (e) PIM-1 protein expression levels were determined in 61 pairs of HCC specimens by western blot, and $\beta$-actin was used as a loading control. The data are presented as the T/N ratio. (f) The correlation of miR-214 and PIM-1 in 40 pairs HCC specimens was analyzed by the Spearman's rank correlation coefficient. (g) The PIM-1 and TR protein expressions are shown in 10 pairs of representative HCC specimens. (h) The cartoon illustrating the major observations reports that the thyroid hormone suppresses HCC cell proliferation by inducing miR-214 to repress PIM-1. $(* \mathrm{p}<0.05 ; * \mathrm{p}<0.01 ; * * \mathrm{p}<0.001$ vs. Vector or Normal). 
In conclusion, miR-214 was directly stimulated by $\mathrm{T}_{3}$ when its receptor bound to the upstream TRE in hepatoma cells. $\mathrm{T}_{3}$ suppressed proliferation by targeting miR-214 to downregulate PIM-1 in hepatoma cells. However, the reduced expression of TR led to the inhibition of miR-214 and, in turn, increased the expression of PIM-1 to promote tumorigenesis.

\section{Methods}

Cell culture and $\mathbf{T}_{3}$ treatment. Human hepatoma cell lines, HepG2, Huh7 and SK-Hep1, were routinely grown in DMEM supplemented with $10 \%$ fetal bovine serum. The HepG2 cell line used in this study was stably transfected with TR $\alpha$ (HepG2-TR $\alpha 1)$ or TR $\beta$ (HepG2-TR $\beta 1$ ). HepG2-neo was used as the control cell line. The serum was depleted of $\mathrm{T}_{3}(\mathrm{Td})$ via a resin treatment. The cells were cultured at $37^{\circ} \mathrm{C}$ in a humidified atmosphere of $95 \%$ air and $5 \% \mathrm{CO}_{2}$.

Establishment of miR-214 overexpressing and knockdown cells. The miR-214-expressing plasmid was cloned into the expression vector pcDNA6.2 followed by transfection into Huh7 cells using the TurboFect reagent (Thermo Fisher Scientific, Waltham, MA, USA). After $48 \mathrm{hrs}$ of incubation, the cells were transferred to medium containing blasticidin for selection. After 2 weeks, the specific overexpression of miR-214 was confirmed via qRT-PCR. The lentivirus, miRZip-214, was purchased from System Biosciences (Mountain View, CA, USA). The plasmid and lentiviral package plasmids were cotransfected into Her-293T cells using the TurboFect reagent kit (Thermo Fisher Scientific) and produced viruses in the cells. After 24 hrs, the viral soup was collected for the infection of the HepG2-TR and SK-Hep1 cells. After 48 hrs of incubation, the cells were transferred into medium containing puromycin for selection. Specific anti-sense miR-214 was confirmed using qRT-PCR.

Real-time polymerase chain reaction. Total RNA was purified using TRIzol reagent (Life Technologies Inc., Carlsbad, CA, USA) according to the supplier's protocol, and cDNA was synthesized using a Superscript II kit (Life Technologies, Karlsruhe, Germany). The miRNA-specific stem-loop RT primers (miR-214-3p: CTCAACTGGTGTCGTGGAGTCGGCAATTC AGTTGAGCTGCCTGTCT;miR-199a-3p:CTCAACTGGTG TCGTGGAGTCGGCAATTCAGTTGAGTAACCAAT), dNTPs (10 nM), Superscript III reverse transcriptase and $1 \mu \mathrm{g}$ total RNA were used for the microRNA RT reaction. All the reactions were conducted in an ABI PRISM 7500 sequencer (Applied Biosystems, Foster City, CA, USA).

Promoter luciferase reporter analysis. Fragments of the miR-214 promoter were inserted into the PA3TK vector (Promega Corp, Madison, WI). To assess the influence of $\mathrm{T}_{3}$ on the transcriptional activity of the miR-214 promoter, HepG2-TR $\alpha 1$ cells were transfected with the miR-214 promoter using the TurboFect (Thermo Fisher Scientific) protocol. Twenty-four hours after the transfection, the cells were treated with $\mathrm{Td} / \mathrm{T}_{3}$. Both the treated and untreated cells were incubated for a further $24 \mathrm{hrs}$ and were lysed to measure the luciferase activity. Luciferase activity was assayed with a luminometer (LMax II 384; Molecular Devices, Sunnyvale, CA, USA).

Chromatin immunoprecipitation (ChIP) assays. The ChIP assay was performed to detect the interactions between the TR and TREs on the miR-214 promoter regions, as previously described ${ }^{67}$.

3'UTR luciferase reporter analysis. Fragments of the PIM-1 $3^{\prime}$ UTR plasmid were inserted into the pMIR-REPORT vector (Applied Biosystems). Serial deletion mutants of the $3^{\prime}$ UTR plasmid were amplified via PCR. To assess the interaction of miR-214 with the PIM-1 3'UTR, Huh7 overexpressing miR-214 cells were co-transfected with the PIM-1 3'UTR (WT and Mutant) and beta-galactosidase. At 24 hrs after the transfection, the cells were incubated for another $24 \mathrm{hrs}$ and were lysed to measure the luciferase activity. Luciferase activity was assayed with a luminometer (LMax II 384; Molecular Devices, Sunnyvale, CA, USA).

Cell proliferation and colony assays. The proliferation capacity of the hepatoma cell lines, under different the conditions, was assessed with the aid of the proliferation and colony assays. The final cell seed number was $3 \times 10^{4}$ cells for the proliferation assay and $3 \times 10^{3}$ cells for the colony assay. The cells were routinely grown in DMEM supplemented with $10 \%$ fetal bovine serum. For the proliferation assay, the cell cultures were treated with trypsin, and the detached cells were counted with a hemocytometer. The colonies were fixed and stained with crystal violet after two weeks.

Mouse xenograft model. miR-214 overexpressing and control Huh7 cells $\left(3 \times 10^{6}\right.$ cells/200 $\left.\mu \mathrm{l}\right)$ were injected subcutaneously into the flanks of immunodeficient nude mice. All the procedures were carried out in accordance with the Guide for the Care and Use of Laboratory Animals issued by the Institutional Animal Care and Use Committee of Chang Gung University and the National Institutes of Health of United States. All methods were approved by the Chang-Gung Institutional Animal Care and Use Committee.

Statistical Analysis. The statistical analyses were conducted using t-tests, a one-way analysis of variance (ANOVA), and a Tukey's honest significant difference post hoc test. The correlation analysis was conducted using spearman. The data are presented as the means \pm standard deviations.

\section{References}

1. Chi, H. C., Chen, C. Y., Tsai, M. M., Tsai, C. Y. \& Lin, K. H. Molecular functions of thyroid hormones and their clinical significance in liver-related diseases. BioMed research international 2013, 601361, https://doi.org/10.1155/2013/601361 (2013).

2. Cheng, S. Y. Multiple mechanisms for regulation of the transcriptional activity of thyroid hormone receptors. Reviews in endocrine \& metabolic disorders 1, 9-18 (2000) 
3. Brent, G. A. Mechanisms of thyroid hormone action. The Journal of clinical investigation 122, 3035-3043, https://doi.org/10.1172/ JCI60047 (2012).

4. Gonzalez-Sancho, J. M., Garcia, V., Bonilla, F. \& Munoz, A. Thyroid hormone receptors/THR genes in human cancer. Cancer letters 192, 121-132 (2003).

5. Cable, E. E. et al. Reduction of hepatic steatosis in rats and mice after treatment with a liver-targeted thyroid hormone receptor agonist. Hepatology 49, 407-417, https://doi.org/10.1002/hep.22572 (2009).

6. Naehrlich, L., Dorr, H. G., Bagheri-Behrouzi, A. \& Rauh, M. Iodine deficiency and subclinical hypothyroidism are common in cystic fibrosis patients. Journal of trace elements in medicine and biology: organ of the Society for Minerals and Trace Elements 27, 122-125, https://doi.org/10.1016/j.jtemb.2012.08.002 (2013).

7. Chi, H. C. et al. Thyroid hormone receptor inhibits hepatoma cell migration through transcriptional activation of Dickkopf 4. Biochemical and biophysical research communications 439, 60-65, https://doi.org/10.1016/j.bbrc.2013.08.028 (2013).

8. Lin, Y. H. et al. Thyroid hormone suppresses cell proliferation through endoglin-mediated promotion of p21 stability. Oncogene 32, 3904-3914, https://doi.org/10.1038/onc.2013.5 (2013).

9. Lin, K. H., Shieh, H. Y., Chen, S. L. \& Hsu, H. C. Expression of mutant thyroid hormone nuclear receptors in human hepatocellular carcinoma cells. Molecular carcinogenesis 26, 53-61 (1999).

10. Davis, F. B. et al. Acting via a cell surface receptor, thyroid hormone is a growth factor for glioma cells. Cancer research 66, 7270-7275, https://doi.org/10.1158/0008-5472.CAN-05-4365 (2006).

11. Plateroti, M., Kress, E., Mori, J. I. \& Samarut, J. Thyroid hormone receptor alphal directly controls transcription of the beta-catenin gene in intestinal epithelial cells. Molecular and cellular biology 26, 3204-3214, https://doi.org/10.1128/MCB.26.8.3204-3214.2006 (2006).

12. Lin, H. Y. et al. Thyroid hormone is a MAPK-dependent growth factor for thyroid cancer cells and is anti-apoptotic. Steroids $\mathbf{7 2}$, 180-187, https://doi.org/10.1016/j.steroids.2006.11.014 (2007).

13. Ambros, V. The functions of animal microRNAs. Nature 431, 350-355, https://doi.org/10.1038/nature02871 (2004).

14. Meister, G. \& Tuschl, T. Mechanisms of gene silencing by double-stranded RNA. Nature 431, 343-349, https://doi.org/10.1038/ nature 02873 (2004).

15. Etheridge, A., Lee, I., Hood, L., Galas, D. \& Wang, K. Extracellular microRNA: a new source of biomarkers. Mutation research 717, 85-90, https://doi.org/10.1016/j.mrfmmm.2011.03.004 (2011).

16. Desvignes, T., Contreras, A. \& Postlethwait, J. H. Evolution of the miR199-214 cluster and vertebrate skeletal development. RNA biology 11, 281-294, https://doi.org/10.4161/rna.28141 (2014).

17. Yang, H. et al. MicroRNA expression profiling in human ovarian cancer: miR-214 induces cell survival and cisplatin resistance by targeting PTEN. Cancer research 68, 425-433, https://doi.org/10.1158/0008-5472.CAN-07-2488 (2008).

18. Scapoli, L. et al. MicroRNA expression profiling of oral carcinoma identifies new markers of tumor progression. International journal of immunopathology and pharmacology 23, 1229-1234 (2010).

19. Orso, F. et al. miR-214 and miR-148b Targeting Inhibits Dissemination of Melanoma and Breast Cancer. Cancer research 76, 5151-5162, https://doi.org/10.1158/0008-5472.CAN-15-1322 (2016).

20. Shih, T. C. et al. MicroRNA-214 downregulation contributes to tumor angiogenesis by inducing secretion of the hepatoma-derived growth factor in human hepatoma. Journal of hepatology 57, 584-591, https://doi.org/10.1016/j.jhep.2012.04.031 (2012).

21. Liu, M. et al. Association of serum microRNA expression in hepatocellular carcinomas treated with transarterial chemoembolization and patient survival. PloS one 9, e109347, https://doi.org/10.1371/journal.pone.0109347 (2014).

22. Xie, Y. \& Bayakhmetov, S. PIM1 kinase as a promise of targeted therapy in prostate cancer stem cells. Molecular and clinical oncology 4, 13-17, https://doi.org/10.3892/mco.2015.673 (2016).

23. Narlik-Grassow, M. et al. The essential role of PIM kinases in sarcoma growth and bone invasion. Carcinogenesis 33, 1479-1486, https://doi.org/10.1093/carcin/bgs176 (2012).

24. Malinen, M. et al. Proto-oncogene PIM-1 is a novel estrogen receptor target associating with high grade breast tumors. Molecular and cellular endocrinology 365, 270-276, https://doi.org/10.1016/j.mce.2012.10.028 (2013).

25. Santio, N. M. et al. The PIM1 kinase promotes prostate cancer cell migration and adhesion via multiple signalling pathways. Experimental cell research 342,113-124, https://doi.org/10.1016/j.yexcr.2016.02.018 (2016).

26. Chiang, W. F. et al. Up-regulation of a serine-threonine kinase proto-oncogene Pim-1 in oral squamous cell carcinoma. International journal of oral and maxillofacial surgery 35, 740-745, https://doi.org/10.1016/j.ijom.2006.01.027 (2006).

27. Peltola, K. et al. Pim-1 kinase expression predicts radiation response in squamocellular carcinoma of head and neck and is under the control of epidermal growth factor receptor. Neoplasia 11, 629-636 (2009).

28. Peng, Y. H. et al. Expression of pim-1 in tumors, tumor stroma and tumor-adjacent mucosa co-determines the prognosis of colon cancer patients. PloS one 8, e76693, https://doi.org/10.1371/journal.pone.0076693 (2013).

29. Yan, B. et al. Clinical and therapeutic relevance of PIM1 kinase in gastric cancer. Gastric cancer: official journal of the International Gastric Cancer Association and the Japanese Gastric Cancer Association 15, 188-197, https://doi.org/10.1007/s10120-011-0097-2 (2012).

30. Ouhtit, A., Muzumdar, S., Gupta, I., Shanmuganathan, S. \& Tamimi, Y. Understanding the functional discrepancy of Pim-1 in cancer. Frontiers in bioscience 7, 208-214 (2015).

31. Merkel, A. L., Meggers, E. \& Ocker, M. PIM1 kinase as a target for cancer therapy. Expert opinion on investigational drugs 21, 425-436, https://doi.org/10.1517/13543784.2012.668527 (2012).

32. Arunesh, G. M., Shanthi, E., Krishna, M. H., Sooriya Kumar, J. \& Viswanadhan, V. N. Small molecule inhibitors of PIM1 kinase: July 2009 to February 2013 patent update. Expert opinion on therapeutic patents 24, 5-17, https://doi.org/10.1517/13543776.2014.84819 6 (2014).

33. Bachmann, M. \& Moroy, T. The serine/threonine kinase Pim-1. The international journal of biochemistry \& cell biology 37, 726-730, https://doi.org/10.1016/j.biocel.2004.11.005 (2005).

34. Magnuson, N. S., Wang, Z., Ding, G. \& Reeves, R. Why target PIM1 for cancer diagnosis and treatment? Future oncology 6, 1461-1478, https://doi.org/10.2217/fon.10.106 (2010).

35. Wang, Y. et al. Downregulation of microRNA33a promotes cyclindependent kinase 6, cyclin D1 and PIM1 expression and gastric cancer cell proliferation. Molecular medicine reports 12, 6491-6500, https://doi.org/10.3892/mmr.2015.4296 (2015).

36. Kim, J. E. et al. A Novel Cinnamon-Related Natural Product with Pim-1 Inhibitory Activity Inhibits Leukemia and Skin Cancer. Cancer research 75, 2716-2728, https://doi.org/10.1158/0008-5472.CAN-14-3655 (2015).

37. Xu, J. et al. PIM-1 contributes to the malignancy of pancreatic cancer and displays diagnostic and prognostic value. Journal of experimental \& clinical cancer research: CR 35, 133, https://doi.org/10.1186/s13046-016-0406-z (2016).

38. Doshi, K. A. et al. Pim kinase inhibition sensitizes FLT3-ITD acute myeloid leukemia cells to topoisomerase 2 inhibitors through increased DNA damage and oxidative stress. Oncotarget 7, 48280-48295, https://doi.org/10.18632/oncotarget.10209 (2016).

39. Chi, H. C. et al. Thyroid hormone protects hepatocytes from $\mathrm{HBx}$-induced carcinogenesis by enhancing mitochondrial turnover. Oncogene, doi:https://doi.org/10.1038/onc.2017.136 (2017).

40. Chi, H. C. et al. Thyroid hormone suppresses hepatocarcinogenesis via DAPK2 and SQSTM1-dependent selective autophagy. Autophagy 12, 2271-2285, https://doi.org/10.1080/15548627.2016.1230583 (2016).

41. Lee, Y. B. et al. Twist-1 regulates the miR-199a/214 cluster during development. Nucleic acids research 37, 123-128, https://doi. org/10.1093/nar/gkn920 (2009). 
42. Hu, J. et al. MiR-215 Is Induced Post-transcriptionally via HIF-Drosha Complex and Mediates Glioma-Initiating Cell Adaptation to Hypoxia by Targeting KDM1B. Cancer cell 29, 49-60, https://doi.org/10.1016/j.ccell.2015.12.005 (2016).

43. Ji, M. et al. The miR-17-92 microRNA cluster is regulated by multiple mechanisms in B-cell malignancies. The American journal of pathology 179, 1645-1656, https://doi.org/10.1016/j.ajpath.2011.06.008 (2011).

44. Wang, P. et al. miR-214/199a/199a* cluster levels predict poor survival in hepatocellular carcinoma through interference with cellcycle regulators. Oncotarget 7, 929-945, https://doi.org/10.18632/oncotarget.6137 (2016).

45. He, G. Y. et al. The FOXD3/miR-214/MED19 axis suppresses tumour growth and metastasis in human colorectal cancer. British journal of cancer 115, 1367-1378, https://doi.org/10.1038/bjc.2016.362 (2016).

46. Lu, Q. et al. miR-214 inhibits invasion and migration via downregulating GALNT7 in esophageal squamous cell cancer. Tumour biology: the journal of the International Society for Oncodevelopmental Biology and Medicine 37, 14605-14614, https://doi. org/10.1007/s13277-016-5320-7 (2016).

47. Chandrasekaran, K. S., Sathyanarayanan, A. \& Karunagaran, D. MicroRNA-214 suppresses growth, migration and invasion through a novel target, high mobility group AT-hook 1, in human cervical and colorectal cancer cells. British journal of cancer 115, 741-751, https://doi.org/10.1038/bjc.2016.234 (2016)

48. Sharma, T., Hamilton, R. \& Mandal, C. C. miR-214: a potential biomarker and therapeutic for different cancers. Future oncology 11, 349-363, https://doi.org/10.2217/fon.14.193 (2015).

49. Xu, Z. \& Wang, T. miR-214 promotes the proliferation and invasion of osteosarcoma cells through direct suppression of LZTS1. Biochemical and biophysical research communications 449, 190-195, https://doi.org/10.1016/j.bbrc.2014.04.140 (2014).

50. Alimirah, F. et al. Crosstalk between the vitamin D receptor (VDR) and miR-214 in regulating SuFu, a hedgehog pathway inhibitor in breast cancer cells. Experimental cell research 349, 15-22, https://doi.org/10.1016/j.yexcr.2016.08.012 (2016).

51. Wang, F., Lv, P., Liu, X., Zhu, M. \& Qiu, X. microRNA-214 enhances the invasion ability of breast cancer cells by targeting p53. International journal of molecular medicine 35, 1395-1402, https://doi.org/10.3892/ijmm.2015.2123 (2015).

52. Xin, R. et al. MicroRNA-214 promotes peritoneal metastasis through regulating PTEN negatively in gastric cancer. Clinics and research in hepatology and gastroenterology 40,748-754, https://doi.org/10.1016/j.clinre.2016.05.006 (2016).

53. Janssen, R. et al. MicroRNA 214 Is a Potential Regulator of Thyroid Hormone Levels in the Mouse Heart Following Myocardial Infarction, by Targeting the Thyroid-Hormone-Inactivating Enzyme Deiodinase Type III. Frontiers in endocrinology 7, 22, https:// doi.org/10.3389/fendo.2016.00022 (2016).

54. Sun, M., Yu, H., Zhang, Y., Li, Z. \& Gao, W. MicroRNA-214 Mediates Isoproterenol-induced Proliferation and Collagen Synthesis in Cardiac Fibroblasts. Scientific reports 5, 18351, https://doi.org/10.1038/srep18351 (2015).

55. Williams, G. R. \& Bassett, J. H. D. Thyroid diseases and bone health. Journal of endocrinological investigation, https://doi.org/10.1007/ s40618-017-0753-4 (2017).

56. Segar, J. L., Volk, K. A., Lipman, M. H. \& Scholz, T. D. Thyroid hormone is required for growth adaptation to pressure load in the ovine fetal heart. Experimental physiology 98, 722-733, https://doi.org/10.1113/expphysiol.2012.069435 (2013).

57. di Giacomo, V. et al. Combined supplementation of ascorbic acid and thyroid hormone T3 affects tenocyte proliferation. The effect of ascorbic acid in the production of nitric oxide. Muscles, ligaments and tendons journal 7, 11-18, https://doi.org/10.11138/ $\mathrm{mltj} / 2017.7 .1 .011$ (2017).

58. Yen, C. C. et al. Mediation of the inhibitory effect of thyroid hormone on proliferation of hepatoma cells by transforming growth factor-beta. Journal of molecular endocrinology 36, 9-21, https://doi.org/10.1677/jme.1.01911 (2006).

59. Liu, X. Y., Liu, Z. J., He, H., Zhang, C. \& Wang, Y. L. MicroRNA-101-3p suppresses cell proliferation, invasion and enhances chemotherapeutic sensitivity in salivary gland adenoid cystic carcinoma by targeting Pim-1. American journal of cancer research $\mathbf{5}$, 3015-3029 (2015).

60. Santio, N. M. et al. Pim Kinases Promote Migration and Metastatic Growth of Prostate Cancer Xenografts. PloS one 10, e0130340, https://doi.org/10.1371/journal.pone.0130340 (2015).

61. Warfel, N. A. \& Kraft, A. S. PIM kinase (and Akt) biology and signaling in tumors. Pharmacology \& therapeutics 151, 41-49, https:// doi.org/10.1016/j.pharmthera.2015.03.001 (2015).

62. Zhang, G., Liu, Z., Cui, G., Wang, X. \& Yang, Z. MicroRNA-486-5p targeting PIM-1 suppresses cell proliferation in breast cancer cells. Tumour biology: the journal of the International Society for Oncodevelopmental Biology and Medicine 35, 11137-11145, https:// doi.org/10.1007/s13277-014-2412-0 (2014).

63. Amatya, V. J., Mawas, A. S., Kushitani, K., Mohi El-Din, M. M. \& Takeshima, Y. Differential microRNA expression profiling of mesothelioma and expression analysis of miR-1 and miR-214 in mesothelioma. International journal of oncology 48, 1599-1607, https://doi.org/10.3892/ijo.2016.3358 (2016).

64. Zhang, Y., Wang, Z. \& Magnuson, N. S. Pim-1 kinase-dependent phosphorylation of p21Cip1/WAF1 regulates its stability and cellular localization in H1299 cells. Molecular cancer research: MCR 5, 909-922, https://doi.org/10.1158/1541-7786.MCR-06-0388 (2007).

65. Zemskova, M., Lilly, M. B., Lin, Y. W., Song, J. H. \& Kraft, A. S. p53-dependent induction of prostate cancer cell senescence by the PIM1 protein kinase. Molecular cancer research: MCR 8, 1126-1141, https://doi.org/10.1158/1541-7786.MCR-10-0174 (2010).

66. Weirauch, U. et al. Functional role and therapeutic potential of the pim-1 kinase in colon carcinoma. Neoplasia 15, 783-794 (2013).

67. Liao, C. H. et al. Positive regulation of spondin 2 by thyroid hormone is associated with cell migration and invasion. Endocrinerelated cancer 17, 99-111, https://doi.org/10.1677/ERC-09-0050 (2010).

\section{Acknowledgements}

This work was supported by grants from Chang Gung Memorial Hospital, Taoyuan, Taiwan (BMRP130, CMRPD1D0381, CMRPD1D0382, CMRPD1D0383; NMRPD1D1021, NMRPD1D1022, and NMRPD1D1023 to KHL, CMRPG6D0013 to CSW) and from the Ministry of Science and Technology of the Republic of China (103-2320-B-182-018-MY3; MOST 104-2811-B-182-011; 105-2811-B-182-011; 105-2314-B182A-044). We would like to thank Taiwan Liver Cancer Network (TLCN) for providing the hepatoma tissue samples and related clinical data (all are anonymous) for our research work.

\section{Author Contributions}

P.S.H., Y.H.L., H.C.C., Y.H.H. designed the study. P.S.H., P.Y.C. performed the experiments. C.T.Y., C.S.W. provided clinical materials. K.H.L. wrote the manuscript with input from all authors.

\section{Additional Information}

Supplementary information accompanies this paper at https://doi.org/10.1038/s41598-017-14864-1.

Competing Interests: The authors declare that they have no competing interests.

Publisher's note: Springer Nature remains neutral with regard to jurisdictional claims in published maps and institutional affiliations. 
(i) Open Access This article is licensed under a Creative Commons Attribution 4.0 International License, which permits use, sharing, adaptation, distribution and reproduction in any medium or format, as long as you give appropriate credit to the original author(s) and the source, provide a link to the Creative Commons license, and indicate if changes were made. The images or other third party material in this article are included in the article's Creative Commons license, unless indicated otherwise in a credit line to the material. If material is not included in the article's Creative Commons license and your intended use is not permitted by statutory regulation or exceeds the permitted use, you will need to obtain permission directly from the copyright holder. To view a copy of this license, visit http://creativecommons.org/licenses/by/4.0/.

(C) The Author(s) 2017 